\title{
Caught Up with the Chordoma: A Rare Extraosseus Encounter
}

\author{
Mohamad Hidir Abdullah*, Ling Jyh Chyang, Neoh Yee Yik and Mohamad Azhari Omar
}

Department of Neurosurgery, Hospital Raja Permaisuri Bainun Ipoh, Perak, Malaysia

*Corresponding author: Mohamad Hidir Abdullah, Department of Neurosurgery, Hospital Raja Permaisuri Bainun Ipoh, Jalan Raja Ashman Shah, 30450 Ipoh, Perak, Malaysia, Tel: +605-2085000

\begin{abstract}
Spine extraosseous chordoma is a rare primary malignant tumor which may be easily misdiagnosed. In this report, we describe a case of an elderly man who presented with severe low back pain and gradual limb weakness with physical examination consistent with L3/L4 radiculopathy. MRI showed intervertebral disc extrusion which is proven histologically as a chordoma. Despite its rarity and radiological finding mimicking the more commonly encountered prolapsed intervertebral disc, a differential diagnosis of spine extraosseous chordoma should be considered and ruled out especially in elderly patient presenting with radiculopathy.
\end{abstract}

\section{Introduction}

Chordoma has been known as a rare slow-growing primary malignant intraosseous tumor with incidence rates between 0.18 to 0.84 per million persons per year [1]. Chordoma originates from notochord remnant [2], therefore can occur along the craniospinal axis. Another spectrum of this disease present without involvement of bone, termed extraosseous chordoma. It has been described in literature occurring in spine, paranasal sinuses, larynx and nasopharynx [3]. Due to its rarity, a spine extraosseous chordoma may be easily neglected and misdiagnosed.

\section{Case Description}

We report a case of an 81-year-old male presenting with severe low back pain (Pain score 8/10) and gradual lower limb weakness until become unable to ambulate. Physical examination is consistent with $L 3 / L 4$ radiculopathy. Pre operatively, power bilateral L2-L3 is MRC 3/5, L4-S1 MRC 4/5 with diminished reflexes and intact sensation.
MRI of lumbosacral spine (Figure 1) showed circumferential disc protrusion at L1/L2, L2/L3, L3/ $L 4$ and L4/L5 level, the most prominent being L3/L4 disc extrusion causing spinal canal stenosis. No bony lytic lesion seen. Disc height is preserved and there is no significant reduction in signal intensity of the disc. A diagnosis of prolapsed intervertebral disc was made with little suspicion of soft tissue tumor.

Patient underwent laminectomy, biopsy and L3L4 short segment instrumentation. Intraoperatively, a whitish mass seen originating from L3/L4 intervertebral disc space exerting mass effect with adherence to dura and dura indentation.

Histopathology examination (Figure 2) showed neoplastic cells with focal nuclear positivity for brachyury, consistent with chordoma.

Post-operative follow up showed significant reduction of pain score to $4 / 10$ however neurology remain similar. Patient was subsequently referred for radiotherapy. In view of elderly patient with poor performance status (KPS $<70$ ) and anticipating poor response to radiation, supportive care and palliation is deemed more appropriate.

\section{Discussion}

Chordoma is a rare slow-growing primary malignant intraosseous tumor with incidence rates between 0.18 to 0.84 per million persons per year [1]. About half of intraosseus chordomas form in the sacrococcygeal region, $30 \%$ in the skull base (usually clivus), while the remaining form in the mobile spine (cervical/thoracic/ lumbar spine) [2]. Chordoma has male predominance, being the only embryonic neoplasm that may occur in 


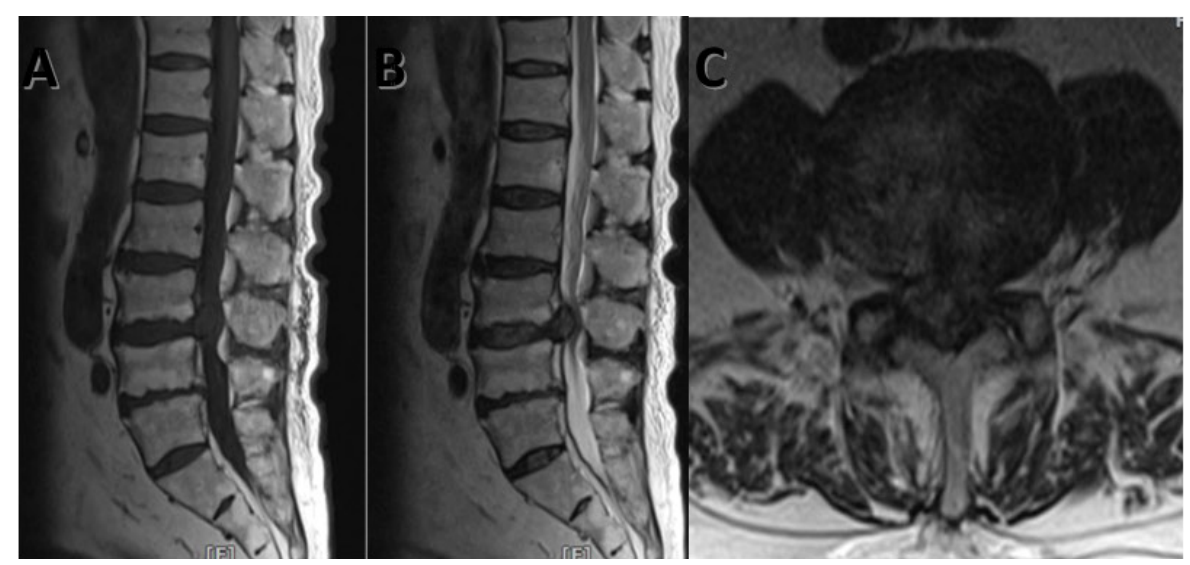

Figure 1: A) Sagittal T1 weighted image; B) Sagittal T2 weighted image revealed extrusion of disc from L3/L4 intervertebral disc causing spinal canal stenosis; C) Axial T2 weighted image at disc level.

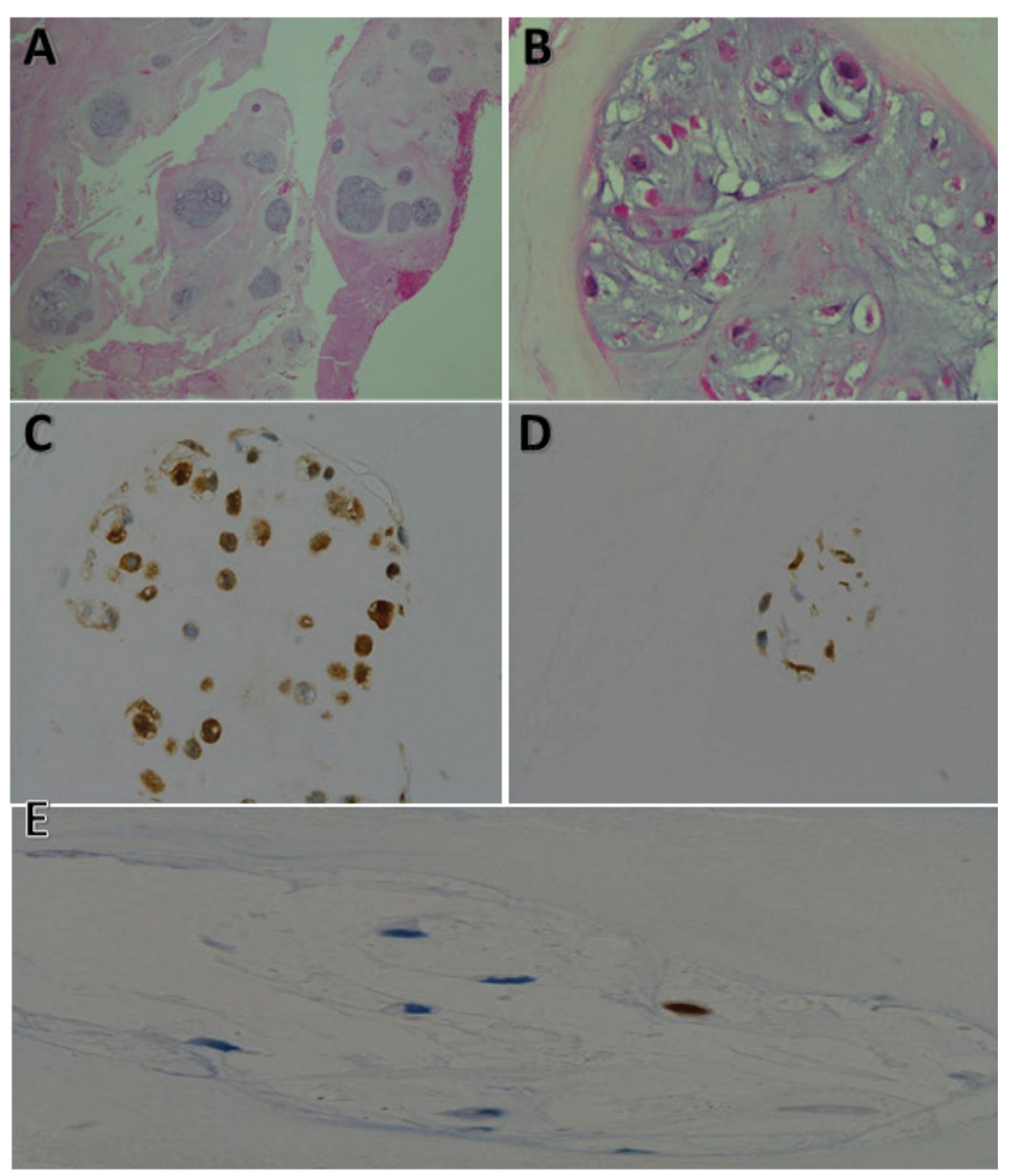

Figure 2: A) x40 magnification: Low power view displaying tumour cells with lobulated architecture and extracellular myxoid matrix; B) x400 magnification: High power view of physaliphorous cells with small nuclei and copious vacuolated cytoplasm; C) x400 magnification: Chordoma cell showing nuclear and cytoplasmic positivity for S100; D) x400 magnification: Chordoma cell showing cytoplasmic positivity for CKAE 1/3; E) Chordoma cell exhibiting focal nuclear staining for Brachyury. 
the later years of life, usually fifth to seven decades [3]. Chordoma may metastasize to the lungs, liver, bones or lymph nodes [4].

Another spectrum of this disease, the extraosseous counterpart can be categorized into epidural and intradural groups. These may be misdiagnosed due to its similarity to the more common neurogenic tumors.

Symptoms are heavily dependent on the site involved and the size of tumor. MRI is imaging of choice to support diagnosis compared to other imaging modalities. Histological examination revealed fibrous tissue separating chords of tumor from mucoid matrix. There are 3 histological subtypes: classical, chondroid and de-differentiated [4]. Immuno-histological markers are positive for nuclear brachyury, epithelial membrane antigen, S-100, cytokeratin, and vimentin [5].

Intraosseous chordoma have high recurrence rate and carries poor prognosis. However, spine extraosseous chordoma is known to be less aggressive with lower recurrence and metastasis rate, and overall carries better prognosis [6]. Current treatment options include surgery, radiotherapy, and targeted therapy $[7,8]$.

\section{Conclusion}

Despite its rarity and radiological finding mimicking a prolapsed intervertebral disc, a differential diagnosis of spine extraosseous chordoma should be considered and ruled out especially in elderly patient presenting with radiculopathy.

\section{Funding}

No funding was received that could have influenced the outcome.

\section{Author's Contribution}

$\mathrm{MH}, \mathrm{LC}, \mathrm{NYY}$ and MA are involved in planning, and development of study concept. NYY and MA are involved with the surgery. MH, LC, NYY and MA contributed to the writing of the manuscript.

\section{References}

1. Bakker $\mathrm{SH}$, Jacobs $\mathrm{WCH}$, Pondaag W, Gelderblom $\mathrm{H}$, Nout RA, et al. (2018) Chordoma: A systematic review of the epidemiology and clinical prognostic factors predicting progression-free and overall survival. Eur Spine J 27: 30433058.

2. Dahlin DC, MacCarty CS (1952) Chordoma: A study of fiftynine case. Cancer 5: 1170-1178.

3. Amendola BE, Amendola MA, Oliver E, McClatchey KD (1986) Chordoma: Role of radiation therapy. Radiology 158: 839-843.

4. Fletcher C, Bridge J, Hogendoorn P, Martens F (2013) World health organization (WHO) classification of tumours of soft tissue and bone. Pathology and Genetics.

5. Crapanzano JP, Ali SZ, Ginsberg MS, Zakowski MF (2001) Chordoma: A cytologic study with histologic and radiologic correlation. Cancer 93: 40-51.

6. Jian Y (2016) Spine Extraosseous chordoma mimicking neurogenic tumors: Report of three cases and review of the literatures. World Journal of Surgical Oncology.

7. Cahide T, Bulut S, Erol FS, Ozercan I, Yildirim H (2002) Chordoma of the thoracic spine: Case report. Neurol Med Chir (Tokyo) 42: 175-180.

8. Noor A, Bindal P, Ramirez M, Vredenburgh J (2020) Chordoma: A case report and review of literature. Am J Case Rep 21: e918927-1-e918927-6. 